\title{
Numerical Study for Near-Bed Variables in Velocity-Skewed Oscillatory Sheet Flow Transport
}

\author{
Xin Chen, ${ }^{1}$ Zichao Zhang, ${ }^{2}$ Yong $\mathrm{Li}^{3}{ }^{3}$ and Xiaoyan Shi ${ }^{1}$ \\ ${ }^{1}$ Beijing Engineering Research Center of Safety and Energy Saving Technology for Water Supply Network System, \\ China Agricultural University, Beijing 100083, China \\ ${ }^{2}$ School of Electric Power, North China University of Water Resources and Electric Power, Zhengzhou 450045, China \\ ${ }^{3}$ Tianjin Centre, China Geological Survey, Tianjin 300170, China
}

Correspondence should be addressed to Xin Chen; chenx@cau.edu.cn

Received 14 April 2017; Revised 2 July 2017; Accepted 11 July 2017; Published 22 August 2017

Academic Editor: Alistair Borthwick

Copyright (C) 2017 Xin Chen et al. This is an open access article distributed under the Creative Commons Attribution License, which permits unrestricted use, distribution, and reproduction in any medium, provided the original work is properly cited.

\begin{abstract}
The near-bed sediment concentration and vertical sediment flux are important in sediment transport mechanics, but they are known much less than the horizontal sediment flux, especially for fine sediment in unsteady flows. A developed two-phase model is applied to study the near-bed sediment concentration, vertical sediment flux, and the relevant total sediment amount for the velocityskewed oscillatory sheet flow transport. With the sediment concentration hindered fall velocity, the classical reference concentration formulas conducted by Engelund and Fredsoe (1976) and Zyserman and Fredsoe (1994) are utilized for the comparison with the twophase model and illustration of the phase-lag and sediment size effects in near-bed sediment concentration and vertical sediment flux. The concentration and vertical flux predicted by the two-phase model agree well with experimental data and are better than empirical formulas. Furthermore, the sediment size effect for pick-up flux function over starved bed is shown to be quite different from that containing sufficient sediment in oscillatory flows.
\end{abstract}

\section{Introduction}

The phenomenon of sediment entrainment and settlement is universal in river and coastal environment and extraordinarily important in the subaquatic geomorphology. The vertical sediment fluxes consist of pick-up flux due to turbulence, settling flux due to gravity, and their summation denoting the variation rate of total sediment amount. Associated with the sediment concentration, they represent the vertical sediment exchange strength and are important for the study of sediment settling down to deposition and picked up into suspension. Most sediment models need a type of nearbed condition associated with vertical sediment flux for bottom boundary conditions. So the predictions of nearbed concentration, vertical sediment flux, pick-up flux, and settling flux are very fundamental for such sediment models.

In fully developed steady flows, the pick-up flux and settling flux are in balance and the total vertical flux is zero. Numerous steady flow formulas have been carried out to predict the vertical sediment flux over loose sediment bed in developed equilibrium steady flows. Generally, some are straight driven by the pick-up function based on clear water flow over starved bed without considering the influence of suspended sediment $[1,2]$, so they are not appropriate in the flow already containing large amount of suspended sediment. The others are evaluated by the balance of pick-up and settling down according to the concentration and sediment fall velocity [3], and the relevant concentration denotes the vertical sediment flux strength.

The near-bed sediment concentration and vertical sediment fluxes in unsteady flows are much more complicated than that in steady flows due to the phase-lag effect, and formulas in unsteady flows are lacking. So the near-bed concentrations denoting vertical flux strength in steady flows [4-6] are applied and widely used in unsteady numerical models for sediment transport [7-9], and even their concentrations drop to zero near the flow reversal when the bottom shear stress is under a threshold. The knowledge of vertical 
sediment flux in unsteady flow is still necessary for numerical models' boundary conditions in engineering.

In unsteady flows, the vertical sediment flux can be derived from the variation rate of sediment amount in movement [10], which can be obtained by single phase models or two-phase models. The single phase numerical models are dependent on the reference concentration or vertical flux as the bottom boundary conditions and cannot describe the vertical flux below reference height. The two-phase models [11-13] can obtain the details of reference concentration and vertical flux as a result of the turbulent upward effect and gravitational settlement. Yu et al. [12] study the vertical flux for medium and coarse sediment, but the model is not valid for fine sediment (diameter $D<0.2 \mathrm{~mm}$ ) when the phase-lag is obvious due to the sediment stress. In addition, the vertical sediment fluxes have not been extracted accurately enough due to the noise or error of modelling high concentration. Very little is known about the near-bed concentration and vertical flux for fine sediment and the phase-lag effects, which are important in the generation of net sediment transport, especially in velocity-skewed oscillatory flows with a sharp wave crest and gentle wave trough.

Present study utilizes a two-phase model to study the near-bed sediment concentration and vertical fluxes in velocity-skewed oscillatory sheet flows containing sufficient amount of sediment. The results mainly exhibit and explain the phase-lag characteristics in concentration and vertical flux at the reference height, near-immobile bed, and very low concentration area for difference sediment sizes.

\section{Applied Models}

Chen and Yu [13] two-phase model is utilized to obtain the sediment concentration and vertical fluxes. For comparison, several steady flow empirical formulas are also applied.

2.1. Two-Phase Flow Model. The two-phase model includes Reynolds averaged continuity and momentum equations:

$$
\begin{aligned}
& \frac{\partial\left[(1-\alpha) u_{f, j}+\alpha u_{s, j}\right]}{\partial x_{j}}=0, \\
& \frac{\partial \alpha}{\partial t}+\frac{\partial\left(\alpha u_{s, j}\right)}{\partial x_{j}}=\frac{\partial}{\partial x_{j}}\left(\frac{v_{s t}}{\delta_{s}} \frac{\partial \alpha}{\partial x_{j}}\right), \\
& \frac{\partial\left[(1-\alpha) u_{f, i}\right]}{\partial t}+\frac{\partial\left[(1-\alpha) u_{f, i} u_{f, j}\right]}{\partial x_{j}} \\
& =-\frac{1-\alpha}{\rho_{f}} \frac{\partial p}{\partial x_{i}}+\frac{\partial \tau_{f j}}{\partial x_{j}}+(1-\alpha) g_{i}-\frac{F_{i}}{\rho_{f}}, \\
& \frac{\partial\left(\alpha u_{s, i}\right)}{\partial t}+\frac{\partial\left(\alpha u_{s, i} u_{s, j}\right)}{\partial x_{j}}=-\frac{\alpha}{\rho_{s}} \frac{\partial p}{\partial x_{i}}+\frac{\partial \tau_{s j}}{\partial x_{j}}+\alpha g_{i}+\frac{F_{i}}{\rho_{s}},
\end{aligned}
$$

where $\alpha$ is the sediment volumetric concentration; $u$ is the velocity; $x$ is the Cartesian coordinate; $t$ is the time; the subscripts $f$ and $s$ stand for the fluid and the sediment phases; the indices $i, j=1,2$ represent horizontal and vertical components and obey summation convention; $v_{s t}$ is the sediment turbulent viscosity confirmed by fluid turbulent viscosity $v_{f t} ; \delta_{s}=1$ is the Schmidt number; $p$ is the pressure; $\tau$ is the stress; $\rho$ is the density; $g$ is the body force; $F$ is the interaction force. $v_{s t}, v_{f t}, \tau$, and $F$ can be seen in Chen and $\mathrm{Yu}$ [13]. Equations (1) are closed by $k-\varepsilon$ turbulent equations:

$$
\begin{aligned}
& \frac{\partial[(1-\alpha) k]}{\partial t}+\frac{\partial\left[(1-\alpha) k u_{f j}\right]}{\partial x_{j}} \\
& =\frac{\partial}{\partial x_{j}}\left[(1-\alpha) v_{f k} \frac{\partial k}{\partial x_{j}}\right]+(1-\alpha)(G-\varepsilon) \\
& \frac{\partial[(1-\alpha) \varepsilon]}{\partial t}+\frac{\partial\left[(1-\alpha) \varepsilon u_{f j}\right]}{\partial x_{j}} \\
& =\frac{\partial}{\partial x_{j}}\left[(1-\alpha) v_{f \varepsilon} \frac{\partial \varepsilon}{\partial x_{j}}\right]+\frac{1-\alpha}{k}\left(C_{1} G \varepsilon-C_{2} \varepsilon^{2}\right),
\end{aligned}
$$

where $k$ is the turbulent kinetic energy (TKE); $\varepsilon$ is the turbulent kinetic energy dissipation rate (DTKE); $v_{f k}=v_{f t}$, $v_{f \varepsilon}=v_{f t} / 1.33, C_{1}=1.44$, and $C_{2}=1.92 ; G$ is the turbulence generation term [13].

Equations (1) and (2) are applied for sheet flows happening at maximum Shields parameter $\theta>0.8-1.0$ when large amounts of sediment are in movement. The computation domain covers the immobile bed and the water tunnel top. The initial bed is located at $x_{2}=0$. The still water condition is assumed to be the initial condition. At the time $t=0$, all flow variables are zero, while $\alpha=0$ at $x_{2}>0$ and $\alpha=0.6$ at $x_{2} \leq 0$.

At the tunnel top, $u_{f, 1}=U(t)=U_{0}\{0.8 \cos [2 \pi(t / T-$ $0.21)]+0.2 \cos [4 \pi(t / T-0.21)]\}$, where $U$ is the free stream (Figure 1); $A=d U / d t ; U_{0}$ and $A_{0}$ are the amplitudes of $U$ and $A ; T$ is the period; subscripts $a$ and $d$ denote acceleration and deceleration stages; scripts $c$ and $t$ denote the crest and trough durations. Positive $(t / T=0-0.42)$ and negative $(t / T=0.42-1)$ $U$ denote onshore and offshore durations, respectively. The vertical gradient of all variables vanished, and the sediment flux is

$$
\frac{v_{s t}}{\delta_{s}} \frac{\partial \alpha}{\partial x_{2}}-u_{s, 2} \alpha=0 .
$$

Nonslip condition is assumed at the bottom that $\alpha=0.6$ and the other variables are set to be zero.

In the lateral boundaries, the horizontal gradients of $\alpha, u$, $k$, and $\varepsilon$ are all zero. The flow is driven by a horizontal pressure gradient:

$$
\frac{\partial p}{\partial x_{1}}=-\rho_{f} \frac{d U}{d t}
$$

2.2. Flux Definition and Empirical Formulas. The sediment amount in movement is defined by Nielsen et al. [10]:

$$
Q\left(x_{2}, t\right)=\int_{x_{2}}^{\infty} \alpha(y, t) d y .
$$




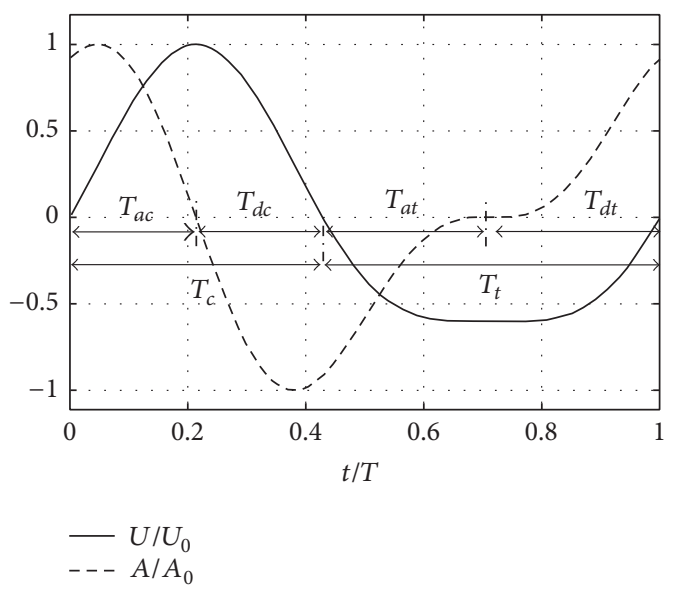

FIGURE 1: Free stream velocity and acceleration.

The total vertical flux is

$$
q_{t}\left(x_{2}, t\right)=\frac{\partial Q\left(x_{2}, t\right)}{\partial t}
$$

and the downward settling flux is

$$
q_{d}\left(x_{2}, t\right)=-\alpha\left(x_{2}, t\right) w\left(x_{2}, t\right),
$$

where $w$ is the fall velocity equal to $-u_{s, 2}$. The pick-up flux is

$$
q_{u}\left(x_{2}, t\right)=q_{t}\left(x_{2}, t\right)-q_{d}\left(x_{2}, t\right) .
$$

The dimensionless equations (10)-(12) are $\Phi=q[(S-$ 1) $\left.g D^{3}\right]^{-0.5}$, where the specific gravity $S=2.65$. In the twophase model, $\alpha$ and $w$ are straight given by (1), and $q_{u}=$ $-v_{s t} / \delta_{s}\left(\partial \alpha / \partial x_{2}\right)$.

For $q_{d}$ comparison, the classical Engelund and Fredsoe [4] formula (EF76) and Zyserman and Fredsoe [5] formula (ZF94) for reference $\alpha$ defined at $x_{2} / D=2$ and $w=w_{0}(1-\alpha)^{n}$ are applied. $w_{0}$ is the single sediment fall velocity in still water [14] and $n$ is the same as Yu et al. [12]. The EF76 formula is

$$
\alpha(2 D, t)=\frac{0.6}{(1+\lambda)^{3}},
$$

$\lambda$

$$
=\kappa c_{r} \sqrt{\frac{\theta-\theta_{c}}{0.013 S \theta}\left\{1-\frac{\pi \mu}{6}\left[\left(\theta-\theta_{c}\right)^{4}+\left(\frac{\pi \mu}{6}\right)^{4}\right]^{-1 / 4}\right\}},
$$

where $D$ is the sediment diameter; $\lambda$ is the linear concentration; $\kappa=0.4$ is the Karman constant; $c_{r}=2$ is associated with the bed roughness; $\theta_{c}=0.05$ is the critical Shields parameter; $\mu=0.6$ is the dynamic friction coefficient. The ZF94 formula is

$$
\alpha(2 D, t)=\frac{0.331\left(\theta-\theta_{c}\right)^{1.75} c_{m}}{c_{m}+0.331\left(\theta-\theta_{c}\right)^{1.75}}
$$

where $c_{m}=0.46$. Figure 2 shows the reference $\alpha$ against $\theta$ for the two empirical formulas. The EF76 considers the dispersive

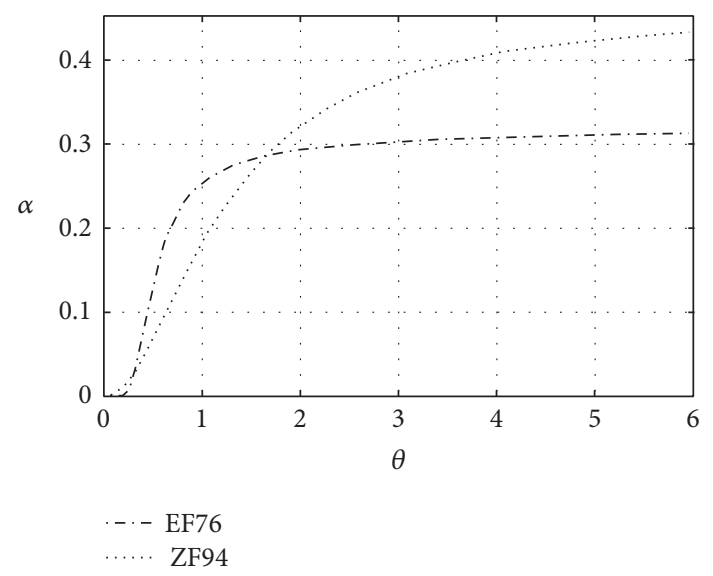

Figure 2: Reference $\alpha$ against $\theta$ in steady flow.

TABLE 1: Computation conditions.

\begin{tabular}{lcccc}
\hline Test case & $U_{0}$ & $D(\mathrm{~mm})$ & $T(\mathrm{~s})$ & $w_{0}(\mathrm{~m} / \mathrm{s})$ \\
\hline Fine: FA5010 & 1.5 & 0.13 & 5.0 & 0.0126 \\
Medium: MA5010 & 1.5 & 0.27 & 5.0 & 0.0387 \\
Coarse: CA5010 & 1.5 & 0.46 & 5.0 & 0.0672 \\
\hline
\end{tabular}

stress from suspended sediment and drag of moving bed, so the reference $\alpha$ is mildly increased when $\theta>1$ and is hindered mildly to be a constant. ZF94 is based on Garcia and Parker's [3] prohibiting a particular trend toward a limitation for high sediment concentration. So the ZF94 also has a limitation of $\alpha$ and keeps a mild increment after $\theta>6$ (Figure 2).

For $q_{u}$ comparison, van Rijn [1] pick-up function over a starved bed is applied:

$$
\begin{aligned}
\Phi_{u} & =\frac{q_{u}}{\sqrt{(S-1) g D^{3}}} \\
& =3.3 \times 10^{-4}\left(\frac{\theta}{\theta_{c}}-1\right)^{1.5}\left[\frac{(S-1) g D^{3}}{v_{f 0}^{2}}\right]^{0.1},
\end{aligned}
$$

where $v_{f 0}$ is the fluid molecular viscosity. $q_{d}$ in EF76 and ZF94 formulas and $q_{u}$ in van Rijn [1] drop to zero when $\theta<\theta_{c}$ without phase-lag effect.

\section{Results}

Computations are based on the O'Donoghue and Wright [15] experiments in Table 1, where the phase-lag effect increases with the decrement of $D$. Results are shown for the classical reference height $x_{2} / D=2$ first and second the near-immobile bed and low concentration areas.

3.1. Reference Vertical Flux. The references $\alpha$ and $\Phi_{d}$ are shown in Figure 3. The absolute locations of $x_{2} / D=2$ are slightly different and are very close to each other, so they can be treated as an approximation to the initial bed. The $\alpha$ distribution pivot, around which $\alpha$ is almost constant in a wave period, exists at the location a little below the initial bed 


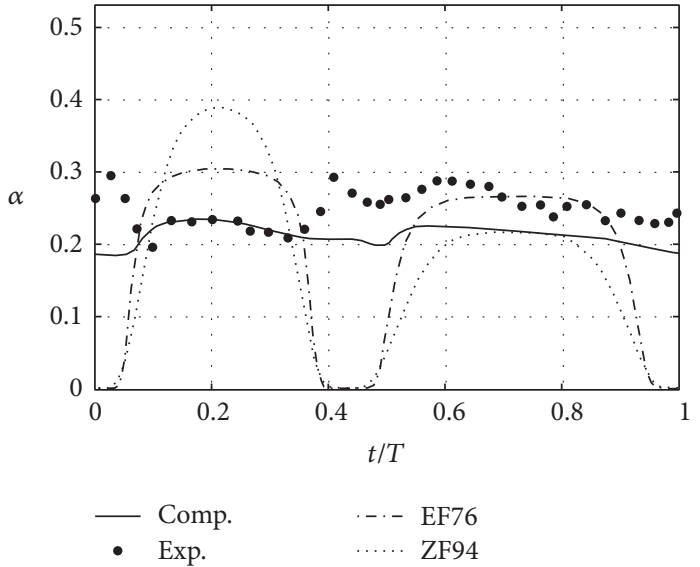

(a) $\alpha$ of FA5010

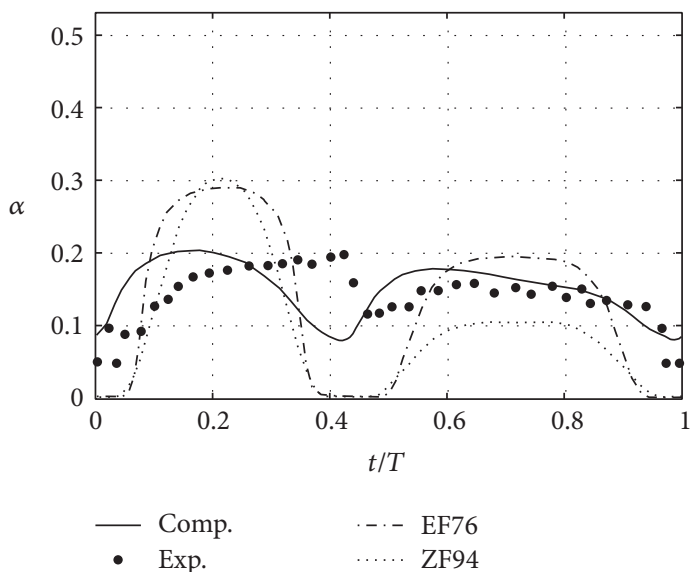

(c) $\alpha$ of MA5010

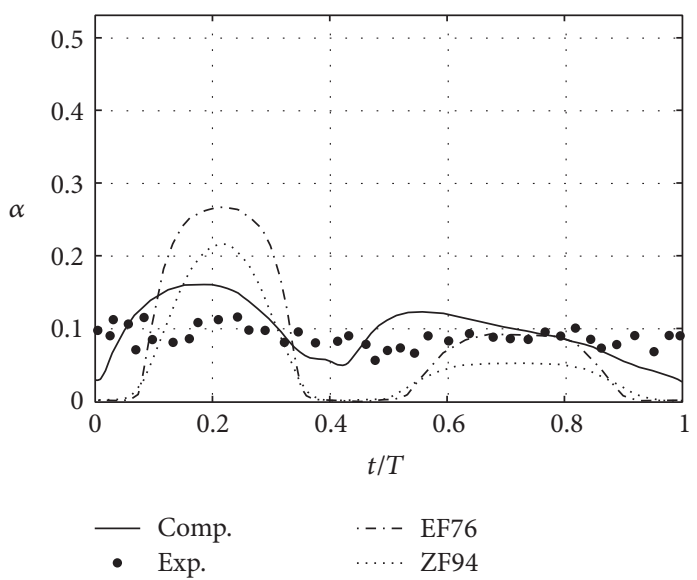

(e) $\alpha$ of CA5010

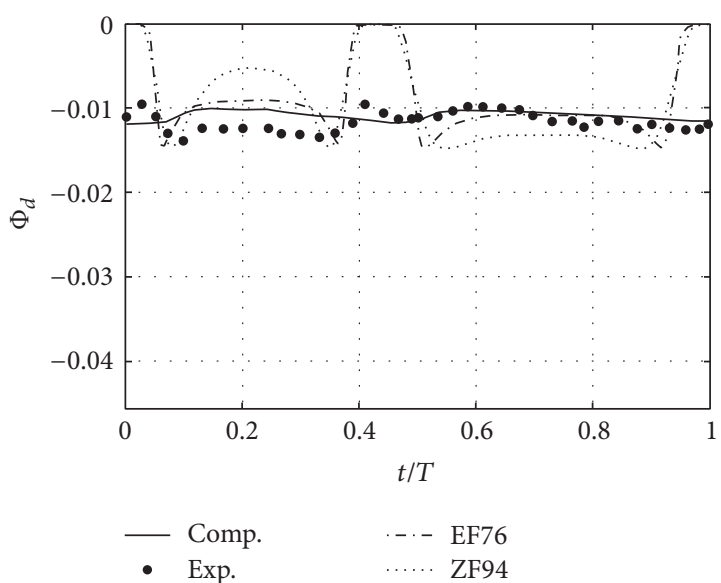

(b) $\Phi_{d}$ of FA5010

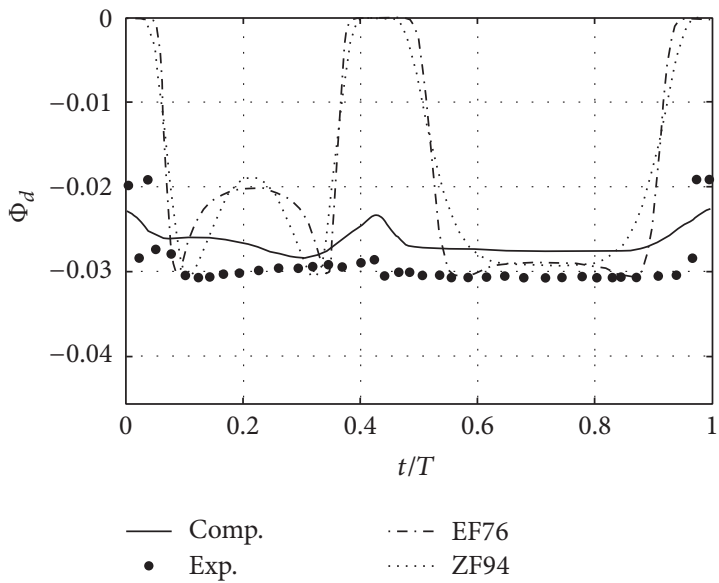

(d) $\Phi_{d}$ of MA5010

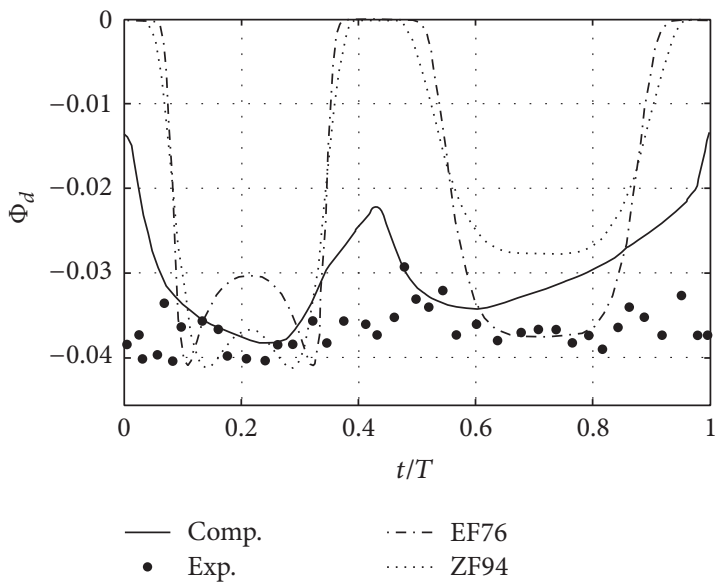

(f) $\Phi_{d}$ of CA5010

Figure 3: Reference $\alpha$ and $\Phi_{d}$.

[15]. In Figures 3(a), 3(c), and 3(e), the location $x_{2} / D=2$ is very close to the pivots, and both the maximum free stream flow and flow reversal would probably lead to $\alpha$ peaks. For the experiment (dot), the $\alpha$ difference between flow crest $(t / T=$ $0.21)$ and flow trough $(t / T=0.71)$ is not obvious and even the crest flow strength is much larger than that at flow trough
(Figure 3), because many sediments picked up at the flow crest are still in movement at the flow trough. Large $D$ corresponds to small concentration magnitude due to the large fall velocity. $\alpha$ never decrease to zero in a period due to the phaselag effect. The results of two-phase model exhibit clearly the decrement of concentration magnitude when $D$ increases, 
TABLE 2: Root-mean-square (RMS) errors at $x_{2} / D=2$.

\begin{tabular}{|c|c|c|c|c|c|c|}
\hline \multirow{2}{*}{ Cases } & \multicolumn{3}{|c|}{$\alpha$} & \multicolumn{3}{|c|}{$\Phi_{d}$} \\
\hline & FA5010 & MA5010 & CA5010 & FA5010 & MA5010 & CA5010 \\
\hline Two-phase model & 0.051 & 0.039 & 0.042 & 0.037 & 0.038 & 0.053 \\
\hline EF76 & 0.138 & 0.094 & 0.086 & 0.091 & 0.100 & 0.118 \\
\hline ZF94 & 0.148 & 0.094 & 0.068 & 0.095 & 0.102 & 0.106 \\
\hline
\end{tabular}

and the computed $\alpha$ is also always above a proper nonzero value (Figures 3(a), 3(c), and 3(e)) due to the phase-lag. Some differences between two-phase model and experimental data exist from a qualitative perspective, probably due to the high concentration measurement uncertainties [15] without mass conservation.

The EF76 and ZF94 are strictly in phase with $U$ without any phase-shift. Mild increment at large shear stress (Figure 2) leads to a small concentration variation near onshore flow peak around $t / T=0.21$ (Figures 3(a) and 3(c)). There are also small concentration variations for EF76 and ZF94 near the offshore flow peak $(t / T=0.6 \sim 0.8)$ caused by the nearly constant $U$ (Figure 1). When $\theta$ is less than a critical level, EF76 and ZF94 predicted $\alpha$ are unrealistic zero (Figure 2), because the formulas are derived without the phase-lags. Predicted concentrations of EF76 and ZF94 increase rapidly in the acceleration stage and decrease fast in the deceleration stage (Figures 3(a), 3(c), and 3(e)). Furthermore, their prediction between onshore and offshore are quite different because of the large different flow strength and bottom shear stress. Although both EF76 and ZF94 can predict the experiments near the flow trough $(t / T=0.6 \sim 0.8)$, they much overestimate the experiments near the flow crest $(t / T=0.21)$. Generally, the EF76 and ZF94 are weaker than the two-phase model results at the flow reversal and flow crest. RMS errors in Table 2 for two-phase model are much less than EF76 and ZF94 formulas.

Corresponding to the concentration due to phase-lag, the experimental $\Phi_{d}$ never decreases to zero near the flow reversal in Figure 3, and the value near the flow crest is close to that near the flow trough. The two-phase model predicted computed $\Phi_{d}$ agree very well with the experiments for nonzero duration and the magnitude increment with $D$. Although the $\alpha$ magnitude decreases with the increment of $D$, the magnitude of $\Phi_{d}$ increases with the increment of $D$ in Figure 3, because the flux is hindered by high concentration at small sediment size. Based on the same $U_{0}=1.5 \mathrm{~m} / \mathrm{s}$, the pick-up flux $\left(\Phi_{u}=-\Phi_{d}\right)$ against $D$ for ZF94 and ZF76 in steady flow is shown in Figure 4, where the dimensionless $D$ is represented by $w_{0} / U_{0}$. The flux increases first and then decreases with $D\left(w_{0} / U_{0}\right)$. The $\Phi_{d}$ magnitude would decrease with small $\alpha$ when $D$ is large enough as the balanced pick-up flux $\left(\Phi_{u}=-\Phi_{d}\right)$ in Figure 4, and finally it decreases to zero when reference $\alpha$ magnitude reduces to zero.

Near the flow crest, there are hollows in EF76 and ZF94 that predicted $\Phi_{d}$ which are far from the experiments (Figures 3(b), 3(d), and 3(f)) with overestimation of $\alpha$ (Figures $3(\mathrm{a}), 3(\mathrm{c})$, and $3(\mathrm{e}))$, because the maximum $\Phi_{d}$ and optimal $\alpha$ exist in the two empirical formulas when we applied $w=w_{0}(1-\alpha)^{n}$, as seen in Figure 5. The maximum $\Phi_{d}$

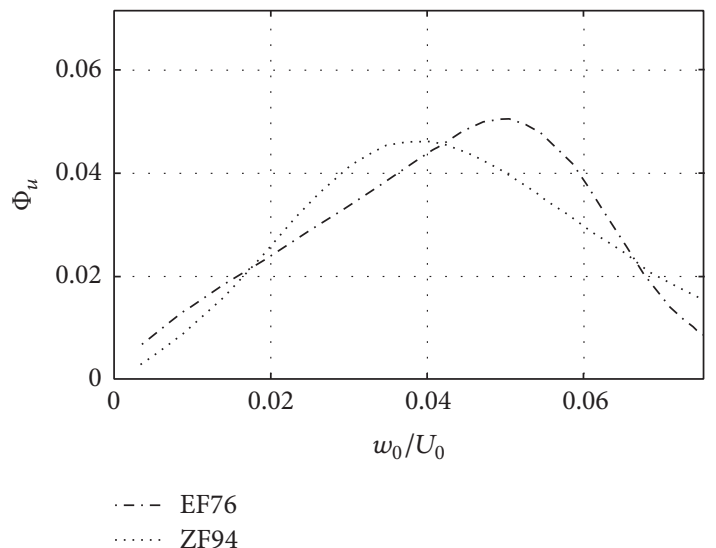

Figure 4: $\Phi_{u}$ in steady flow.

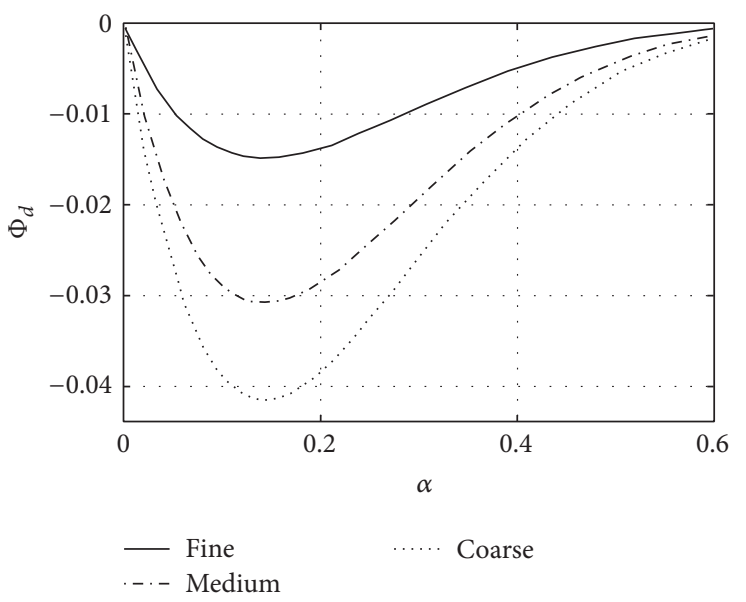

Figure 5: $\Phi_{d}$ of different $D$ in steady flow.

approximately corresponds to optimal $\alpha=0.14$, after which $\Phi_{d}$ decreases with the increment of $\alpha$ by the hindered fall velocity. Near the flow trough, both EF76 and ZF94 can predict $\Phi_{d}$ well due to the agreement of concentration. At flow reversal, both the EF76 and EF94 predicted $\Phi_{d}$ drop to zero because of unrealistic zero $\alpha$ (Figures 3(a), 3(c), and 3(e)) when $\theta$ is less than a critical value. Predicted $\Phi_{d}$ of EF76 and ZF94 changes rapidly near flow reversal with rapid change of $\alpha$. Generally, the RMS errors for EF76 and ZF94 are much larger than two-phase model for $\Phi_{d}$ (Table 2).

Against the settling down induced by gravity, the pickup effect induced by turbulence maintains the suspension of sediment, and the total vertical sediment flux $\left(\Phi_{t}\right)$ is the balance of pick-up flux $\left(\Phi_{u}\right)$ and settling flux $\left(\Phi_{d}\right)$. Figure 6 

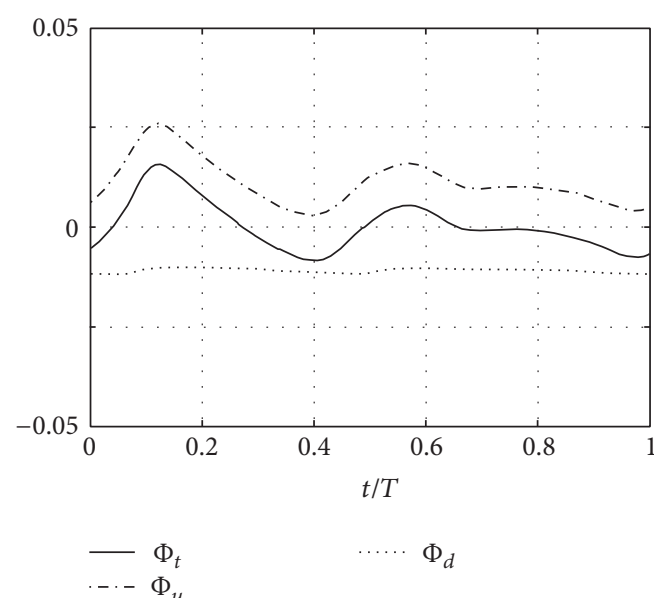

(a) $\Phi$ at $x_{2} / D=2$, FA5010

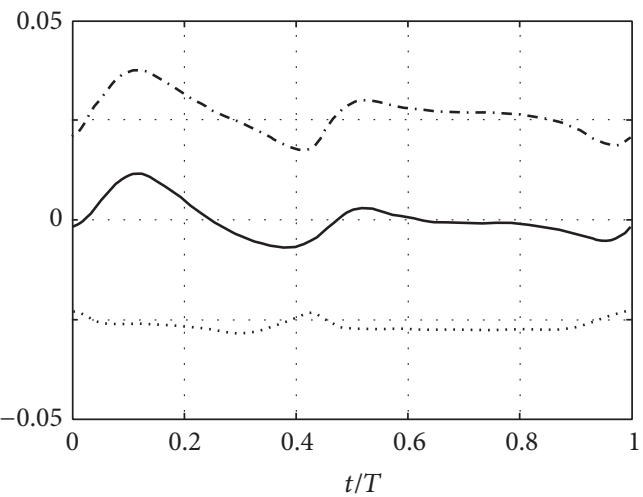

$-\Phi_{t}$

(c) $\Phi$ at $x_{2} / D=2$, MA5010

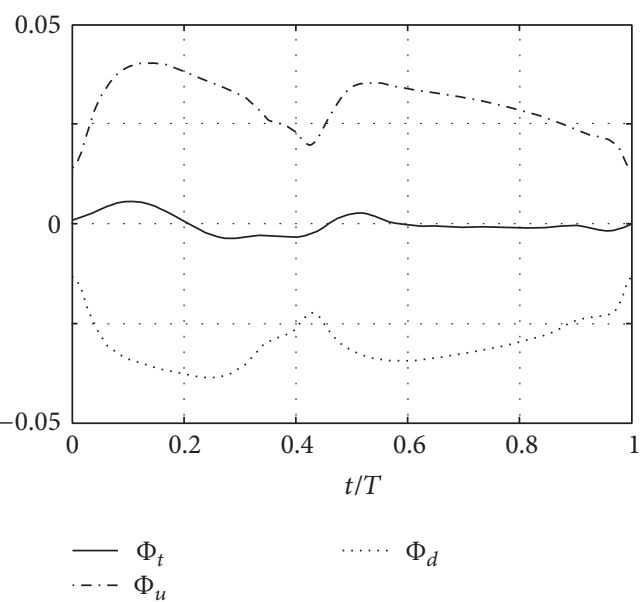

(e) $\Phi$ at $x_{2} / D=2$, CA5010

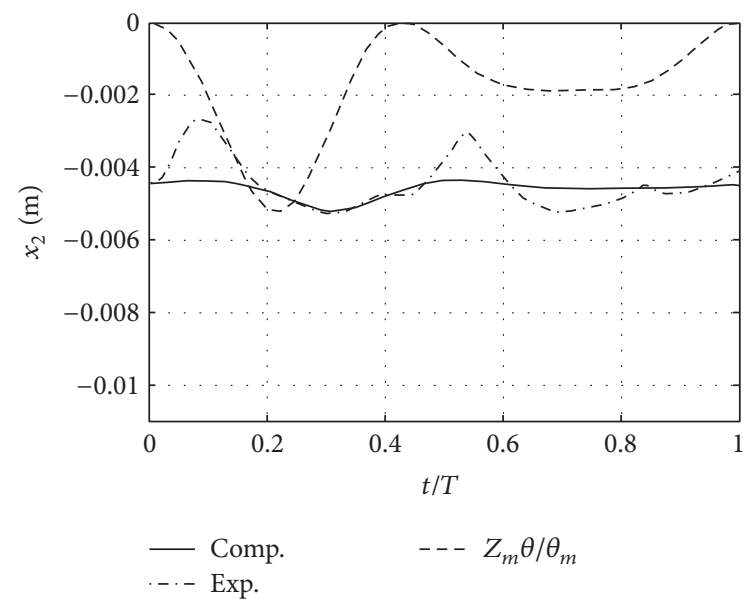

(b) Z, FA5010

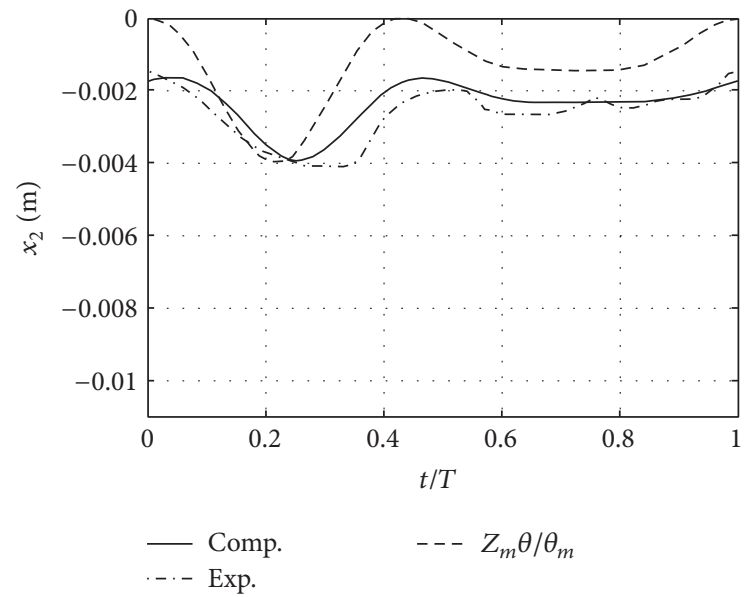

(d) Z, MA5010

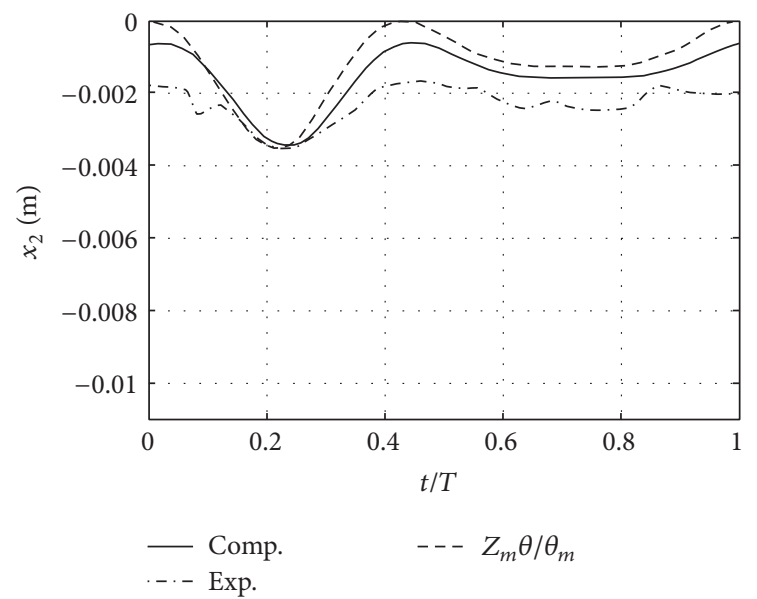

(f) Z, CA5010

Figure 6: Vertical sediment flux and immobile bed surface.

shows the two-phase model computed $\Phi_{t}$ and $\Phi_{u}$ at $x_{2} / D=$ 2 and the immobile bed surface level denoted by $Z$ ( $\alpha=$ 0.594 ). To illustrate their relationships availably and validate $\Phi_{u}$ indirectly, $\Phi_{d}$ in Figure 3 is included again. Magnitudes of $\Phi_{u}$ and $\Phi_{d}$ are around each other with opposite direction, and they are in periodic averaged balance with averaged $\Phi_{t}=0$. As the difference of $\Phi_{u}$ and $\Phi_{d}, \Phi_{t}$ is almost one magnitude smaller than $\Phi_{u}$ and $\Phi_{d} . \Phi_{u}$ increases and reaches a local maximum value before flow crest $(t / T=0.21)$ or flow trough $(t / T=0.71)$ and decreases to a local minimum 
value before flow reversal both in the onshore and in the offshore durations. Correspondingly, $\Phi_{t}$ almost follows the same variation tendency as $\Phi_{u}$. Similar process can be seen in Ribberink et al. [16], Nielsen et al. [10], Li et al. [17], and Bakhtyar et al. [11]. Onshore magnitudes of $\Phi_{u}$ and $\Phi_{t}$ are larger than that at offshore, and the onshore variations are more rapid than offshore.

$Z$ almost varies together with $\Phi_{t}$ which decided the bed morphology in the present three cases in Figure 6. The bed morphology repeats periodically in oscillatory sheet flow conditions without horizontal variation. When $\Phi_{t}>0, Z$ decreases because sediment below the initial bed is picked up. $Z$ decreases to minimum $(t / T=0.25-0.3)$ almost after $\Phi_{t}$ decreases to zero. While $\Phi_{t}<0, Z$ increase because sediment above the initial bed settles down. $Z$ increases to local maximum $(t / T=0.45-0.5)$ almost after $\Phi_{t}$ increases to zero. $Z$ never drops to zero due to the phase-lag effect. For comparison, $Z_{m} \theta / \theta_{m}$ is shown considering theoretical $Z / Z_{m}=\theta / \theta_{m}$ in steady flow, where $Z_{m}$ is the maximum $Z$. The difference between $Z$ and $Z_{m} \theta / \theta_{m}$ denotes the phaselag. There is a time phase-shift between $Z$ and $Z_{m} \theta / \theta_{m}$. For fine $0.13 \mathrm{~mm}$, the phase-lag effect is the largest with the most obvious difference between $Z$ and $Z_{m} \theta / \theta_{m}$, while the phaselag effect for $0.46 \mathrm{~mm}$ is the smallest because present $Z$ almost coincides with $Z_{m} \theta / \theta_{m}$

Corresponding to $\Phi_{d}$, the $\Phi_{u}$ magnitude also increases with the increment of $D$ for the three sizes. But it does not increase with the increment of $D$ monotonously, which is similar to Figure 4. In the two-phase model, $\Phi_{u}$ is obtained from the turbulence diffusion term $-v_{s t} / \delta_{s}\left(\partial \alpha / \partial x_{2}\right)$ modelled by the classical gradient transport assumption. There are two reasons for the smallest $\Phi_{u}$ amplitude with fine $D$ $=0.13 \mathrm{~mm}$ in Figure 6: the concentration gradient and the turbulence intensity. With $D=0.13 \mathrm{~mm}$, much sediment is picked up for suspension and $\partial \alpha / \partial x_{2}$ at $x_{2} / D=2$ becomes smaller than $D=0.27-0.46 \mathrm{~mm}$. When much sediment is already in suspension in the boundary layer, much turbulence energy is dissipated $[15,18]$ corresponding to small $v_{s t}$ and restrains other sediments to be picked up.

For comparison without the effect of suspended hindered sediment, van Rijn [1] pick-up flux function is shown in Figure 7 under the same $U_{0}=1.5 \mathrm{~m} / \mathrm{s}$. van Rijn [1] pickup function decreases with the increment of $D$, which is opposite to the flows containing sufficient sediment amount in Figures 4 and 5. The onshore magnitude is much larger than offshore magnitude without the effect of suspended hindered sediment. In the experiment [15], $\Phi_{u}$ did not drop to zero due to the shear (velocity overshoot) and turbulence that exist near flow reversal. But van Rijn [1] function based on steady flow drops to zero around flow reversal. Comparing the van Rijn pick-up function (Figure 7) with $\Phi_{u}$ given by two-phase model (Figure 6), their $\Phi_{u}$ magnitudes are the closest only for $D=0.46 \mathrm{~mm}$ when the suspension sediment amount is the smallest (Figure 8). Over all, the van Rijn [1] pick-up function is not valid for the oscillatory sheet flow when the maximal $\theta>0.8-1.0$ and sufficient sediment is in suspension.

In developed oscillatory sheet flow, the averaged $\Phi_{t}$ is zero, and suspended sediment is quasi-steady saturated and

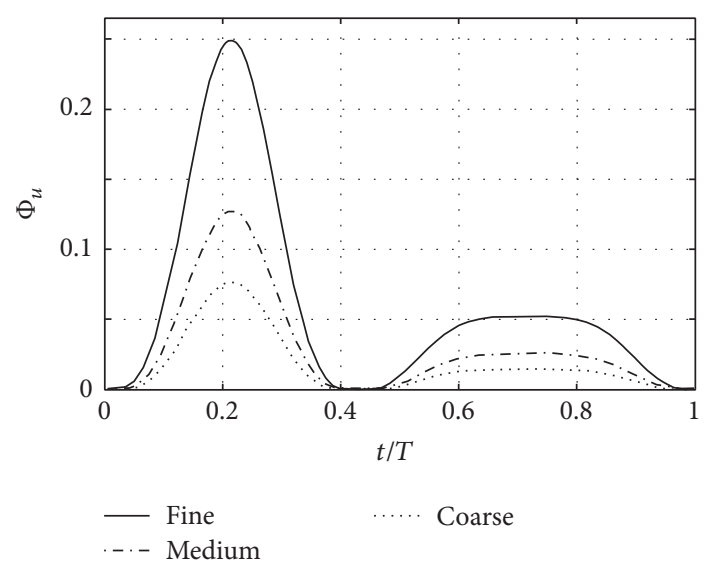

FIGURE 7: Pick-up function of van Rijn.

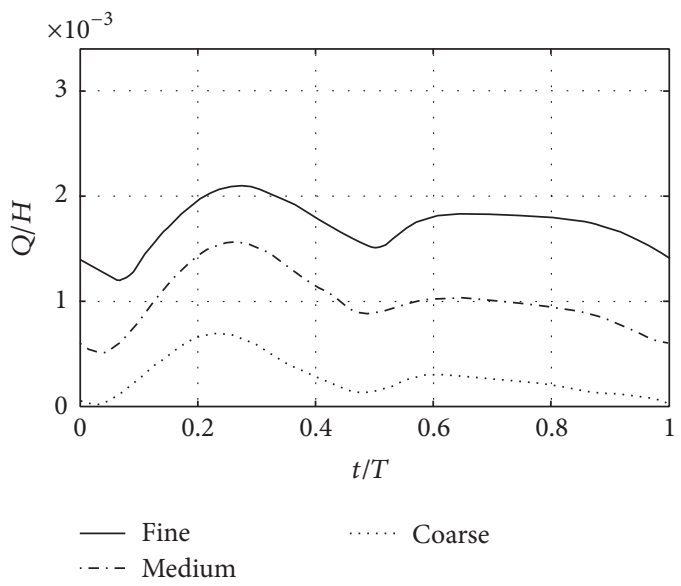

Figure 8: Sediment amount above $x_{2} / D=2$.

hinders the pick-up process. The vertical fluxes display the sediment exchange in vertical direction, but they can not be used as the index for the sediment-laden capacity of oscillatory sheet flow. The sediment amount $Q$ is in a quasisteady status in the developed stage and can be used to estimate the sediment-laden capacity. $Q$ above $x_{2} / D=2$ is shown in Figure 8 , where $H=0.5 \mathrm{~m}$ is the water depth. $Q$ increases with the decrement of $D$. The variation rate of $Q$ is $\Phi_{t}$ (see (6)), so $Q$ reaches a local maximum value when positive $\Phi_{t}$ decreases to zero near the flow peak and reaches a local minimum value when negative $\Phi_{t}$ increases to zero near the flow reversal. Due to large phase-lag, the $Q$ magnitude's ratio between onshore $(t / T=0-0.42)$ and offshore $(t / T=$ $0.42-1$ ) for $0.13 \mathrm{~mm}$ case is 0.87 , much larger than 0.41 for $0.46 \mathrm{~mm}$ case.

3.2. Other Vertical Fluxes. The near-bed flux at the locations above or below the reference height is essential for better understanding of the near-bed sediment exchange. Figure 9 shows the two-phase model computed vertical fluxes at $x_{2}$ $=10 \mathrm{~mm}$ and $x_{2}=-3.5 \mathrm{~mm} .-3.5 \mathrm{~mm}$ is in the main eroded area with high concentration and almost fully hindered $w_{0}$. $10 \mathrm{~mm}$ is out of the sheet flow layer where particle interactions 


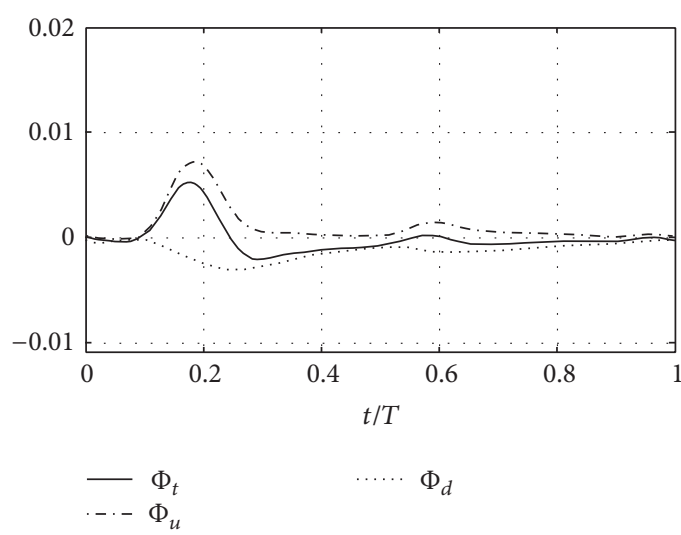

(a) FA5010, $x_{2}=-3.5 \mathrm{~mm}$

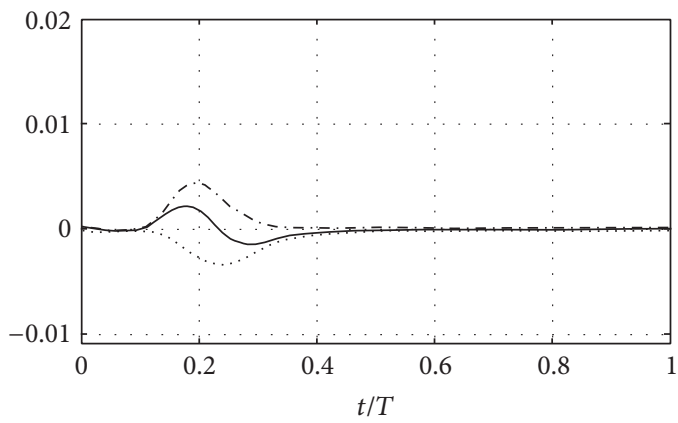

$-\cdot-\Phi_{t}$
$\cdots$

(c) MA5010, $x_{2}=-3.5 \mathrm{~mm}$

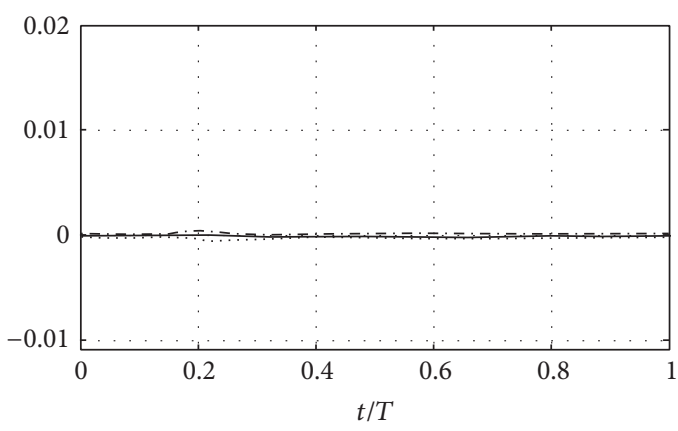

$-\Phi_{t}$

(e) CA5010, $x_{2}=-3.5 \mathrm{~mm}$

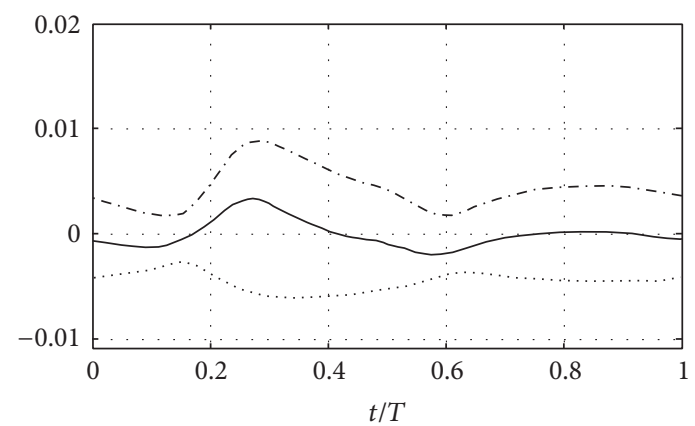

$-\Phi_{t} \quad \cdots \cdots \Phi_{d}$

(b) FA5010, $x_{2}=10 \mathrm{~mm}$
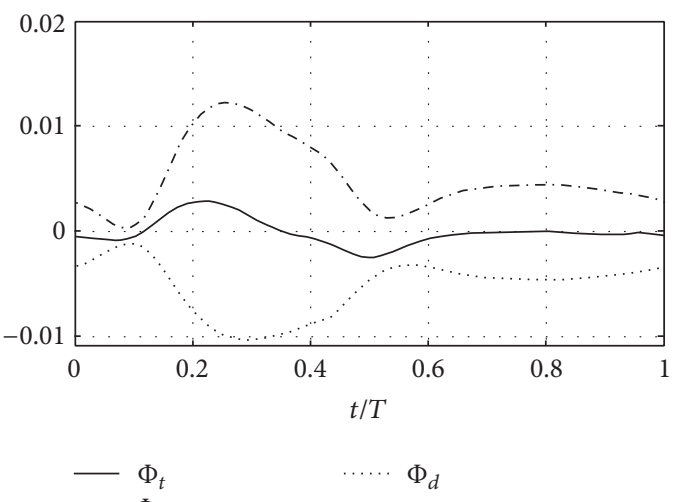

$\cdot-\cdot \Phi_{u}$

(d) MA5010, $x_{2}=10 \mathrm{~mm}$

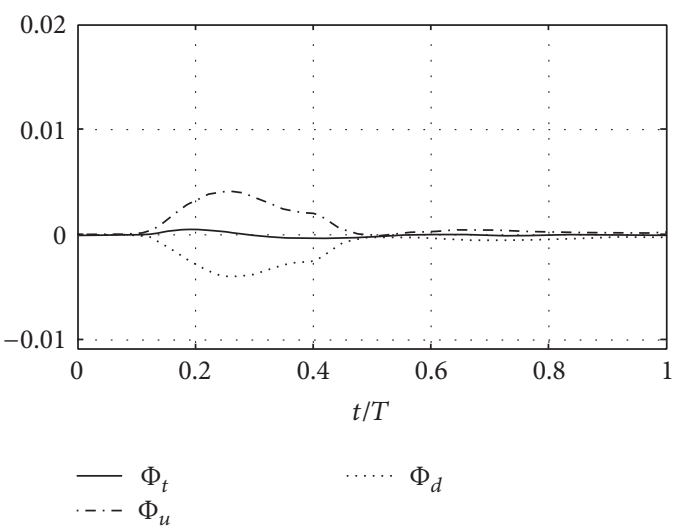

(f) CA5010, $x_{2}=10 \mathrm{~mm}$

Figure 9: Computed vertical flux at $x_{2}=-3.5 \mathrm{~mm}$ and $x_{2}=10 \mathrm{~mm}$.

can be negligible and is still in the oscillatory boundary layer with small concentration and unhindered $w_{0}$. Because the absolute locations of $x_{2} / D$ in the main eroded area or low concentration area for three sizes are far from each other, the vertical locations in Figure 9 are in absolute millimetres rather than as a function of the diameter. At $x_{2}=-3.5 \mathrm{~mm}$, $\Phi_{u}$, $\Phi_{d}$, and $\Phi_{t}$ decrease with the increment of $D$. Two peak values exist in Figure 9(a) and onshore magnitude is larger than offshore, but only one peak value exists near the onshore flow crest in Figure 9(c), while in Figure 9(e) almost zero fluxes exist because $-3.5 \mathrm{~mm}$ is nearly located at the immobile bed for $D=0.46 \mathrm{~mm}$. At $x_{2}=10 \mathrm{~mm}$, obvious phases for all the fluxes are shifted after $x_{2} / D=2$. Both the maximum $\Phi_{u}$ and $\Phi_{d}$ are around 0.01 , which are much larger than $\Phi_{t}$. For fine and medium sediment (Figures 9(b) and 9(d)), their magnitudes of $\Phi_{u}, \Phi_{d}$, and $\Phi_{t}$ are similar to and larger than coarse sediment (Figure 9(f)). For coarse sediment, both $\Phi_{u}$ and $\Phi_{d}$ only exist near the onshore flow peak, and $\Phi_{t}$ almost disappear compared to the fine and medium sediment cases. Comparing the results in Figure 9 to that in Figure 6, all $\Phi_{t}$, 


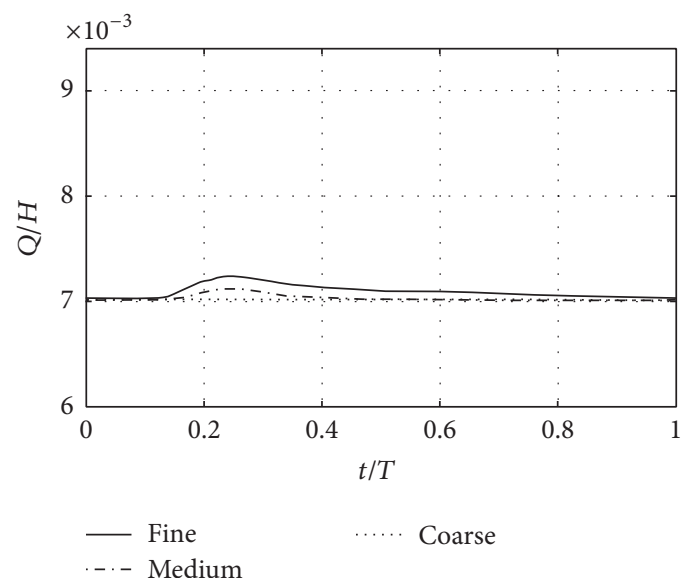

(a) Above $x_{2}=-3.5 \mathrm{~mm}$

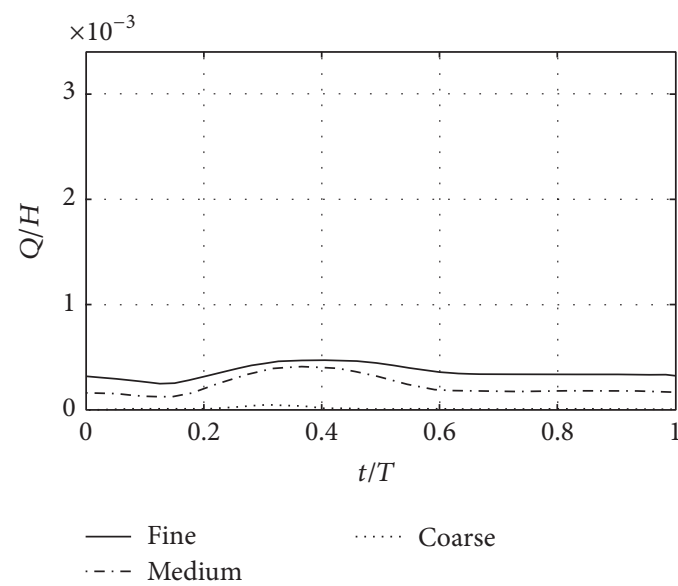

(b) Above $x_{2}=10 \mathrm{~mm}$

FIGURE 10: Sediment amount above $x_{2}=-3.5 \mathrm{~mm}$ and $x_{2}=10 \mathrm{~mm}$.

$\Phi_{u}$, and $\Phi_{d}$ decrease from the initial bed toward the immobile bed and the top of tunnel. The decrement is also seen in Li et al. [17] and Bakhtyar et al. [11]. All the fluxes finally decrease to zero when the location is down into the immobile bed or up to the top of tunnel.

Also, $Q$ above the locations $-3.5 \mathrm{~mm}$ and $10 \mathrm{~mm}$ are shown (Figure 10). At $x_{2}=-3.5 \mathrm{~mm}$ and $x_{2}=-10 \mathrm{~mm}$, the variation amplitudes of each sediment sizes are much smaller than that at $x_{2} / D=2$ (Figure 8) due to smaller $\Phi_{t}$ in Figure 9. Finally, the $Q$ variation amplitudes decrease to zero in the immobile bed or tunnel top corresponding to $\Phi_{t}=0$ (Figure 9).

\section{Conclusions}

The present work studies the near-bed conditions in velocityskewed oscillatory sheet flows with a two-phase model and classical empirical formulas. The phase-lag and sediment size effects in concentration, vertical sediment flux, and sediment amount are focused on the area near the initial bed, the immobile bed, and the low concentration area.

Due to the phase-lag, the reference concentration and the settling flux never drop to zero in a period even at the flow reversal, and the relevant magnitudes between onshore and offshore are similar. The two-phase model computed reference concentration and settling flux by two-phase model agree with experiment very well and are better than empirical formulas. Under the same flows, reference concentration magnitudes decrease with the increment of sediment size due to the increment of fall velocity. While, for settling flux, as the product of concentration and fall velocity, the magnitude increases first and then decreases with the increment of sediment size.

The pick-up flux and settling flux are in periodic averaged balance in oscillatory sheet flow, and their magnitudes are around each other with opposite direction. The pick-up flux increases and reaches a local maximum value before flow peak and decreases to a local minimum value before flow reversal both in the onshore and in the offshore durations.
Corresponding to the settling flux, the pick-up flux also increases first and then decreases with the increment of sediment size.

The sediment amount magnitude increases with the decrement of sediment size. Phase-lag effect increases the sediment amount magnitude's ratio between onshore and offshore. As the sediment amount variation rate, the total vertical flux is much smaller than the pick-up flux and varies almost following the pick-up flux. The vertical sediment flux magnitude decreases from the initial bed toward immobile bed and top of tunnel.

\section{Conflicts of Interest}

The authors declare that they have no conflicts of interest.

\section{Acknowledgments}

This work was supported by the National Natural Science Foundation of China (Grants no. 51609244 and no. 11472156) and National Science-Technology Support Plan of China (Grant no. 2015BAD20B01).

\section{References}

[1] L. C. van Rijn, "Sediment pick-up functions," Journal of Hydraulic Engineering, vol. 110, no. 10, pp. 1494-1502, 1984.

[2] D. Zhong, G. Wang, and Y. Ding, "Bed sediment entrainment function based on kinetic theory," Journal of Hydraulic Engineering, vol. 137, no. 2, pp. 222-233, 2011.

[3] M. Garcia and G. Parker, "Entrainment of bed sediment into suspension," Journal of Hydraulic Engineering, vol. 117, no. 4, pp. 414-435, 1991.

[4] F. Engelund and J. Fredsoe, "A sediment transport model for straight alluvial channels," Nordic Hydrology, vol. 7, pp. 295-324, 1976.

[5] J. A. Zyserman and J. Fredsoe, "Data analysis of bed concentration of suspended sediment," Journal of Hydraulic Engineering, vol. 120, no. 9, pp. 1021-1042, 1994. 
[6] S. Wright and G. Parker, "Flow resistance and suspended load in sand-bed rivers: Simplified stratification model," Journal of Hydraulic Engineering, vol. 130, no. 8, pp. 796-805, 2004.

[7] M. Li, P. T. Fernando, S. Pan, B. A. O'Connor, and D. Chen, "Development of a quasi-3d numerical model for sediment transport prediction in the coastal region," Journal of HydroEnvironment Research, vol. 1, no. 2, pp. 143-156, 2007.

[8] B. G. Ruessink, T. J. J. Van Den Berg, and L. C. Van Rijn, "Modeling sediment transport beneath skewed asymmetric wavesabove a plane bed," Journal of Geophysical Research: Oceans, vol. 114, no. 11, article C11021, 2009.

[9] W. N. M. Hassan and J. S. Ribberink, "Modelling of sand transport under wave-generated sheet flows with a RANS diffusion model," Coastal Engineering, vol. 57, no. 1, pp. 19-29, 2010.

[10] P. Nielsen, K. Van Der Wal, and L. Gillan, "Vertical fluxes of sediment in oscillatory sheet flow," Coastal Engineering, vol. 45, no. 1, pp. 61-68, 2002.

[11] R. Bakhtyar, A. Yeganeh-Bakhtiary, D. A. Barry, and A. Ghaheri, "Two-phase hydrodynamic and sediment transport modeling of wave-generated sheet flow," Advances in Water Resources, vol. 32, no. 8, pp. 1267-1283, 2009.

[12] X. Yu, T.-J. Hsu, J. T. Jenkins, and P. L.-F. Liu, "Predictions of vertical sediment flux in oscillatory flows using a two-phase, sheet-flow model," Advances in Water Resources, vol. 48, pp. 217, 2012.

[13] X. Chen and X. Yu, "A numerical study on oscillatory flowinduced sediment motion over vortex ripples," Journal of Physical Oceanography, vol. 45, no. 1, pp. 228-246, 2015.

[14] L. C. van Rijn, Principles of Sediment Transport in Rivers, Estuaries and Coastal Seas, Aqua Publications, 1993.

[15] T. O’Donoghue and S. Wright, "Concentrations in oscillatory sheet flow for well sorted and graded sands," Coastal Engineering, vol. 50, no. 3, pp. 117-138, 2004.

[16] J. S. Ribberink, C. M. Dohmen-Janssen, D. M. Hanes, S. R. McLean, and C. Vincent, "Near-bed sand transport mechanisms under waves," in Proceedings of the 27th International Conference on Coastal Engineering, ICCE 2000, pp. 3263-3276, Sydney, Australia, July 2000.

[17] M. Li, S. Pan, and B. A. O'Connor, "A two-phase numerical model for sediment transport prediction under oscillatory sheet flows," Coastal Engineering, vol. 55, no. 12, pp. 1159-1173, 2008.

[18] K. C. Wilson, "Friction of wave-induced sheet flow," Coastal Engineering, vol. 12, no. 4, pp. 371-379, 1989. 


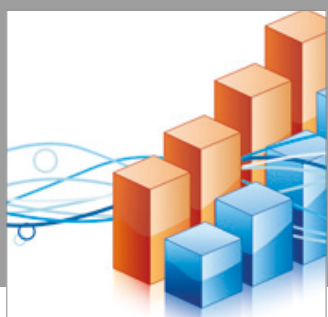

Advances in

Operations Research

vatersals

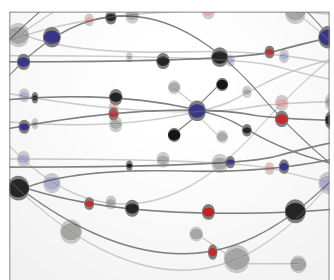

\section{The Scientific} World Journal
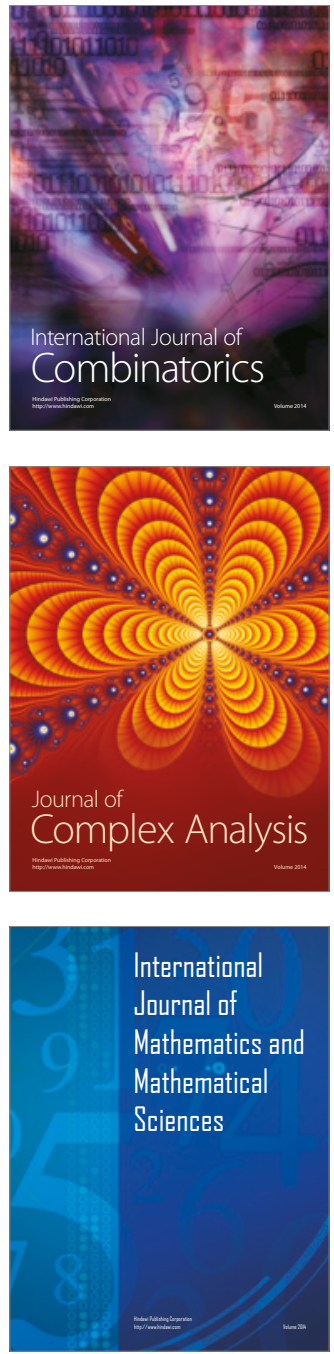
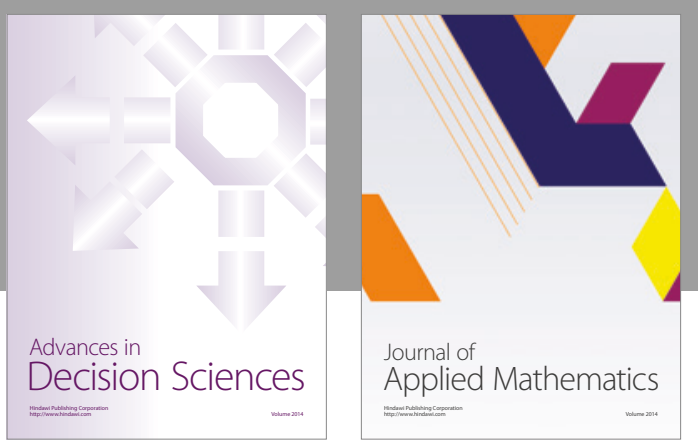

Algebra

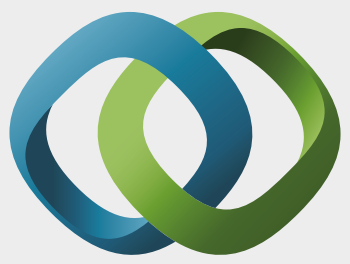

\section{Hindawi}

Submit your manuscripts at

https://www.hindawi.com
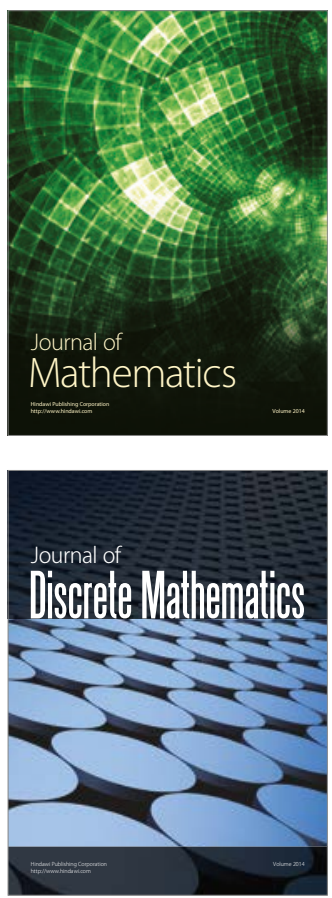

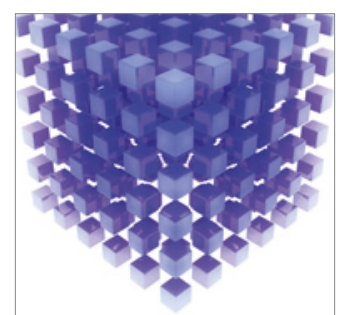

Mathematical Problems in Engineering
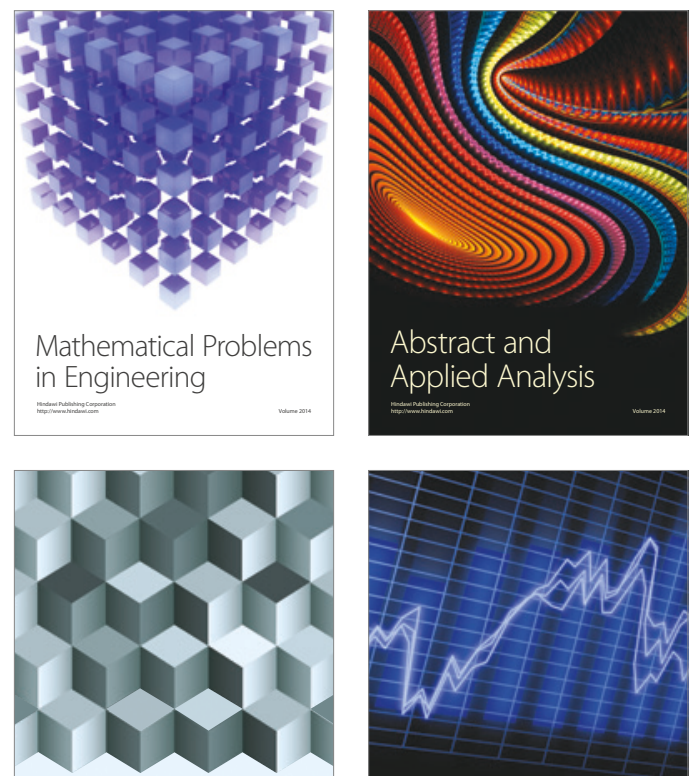

Journal of

Function Spaces

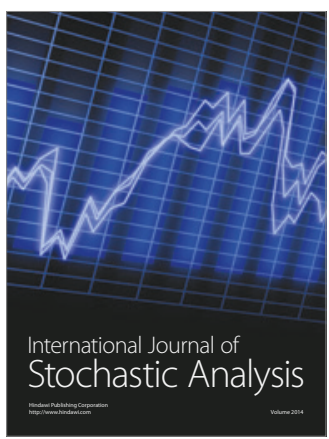

Probability and Statistics
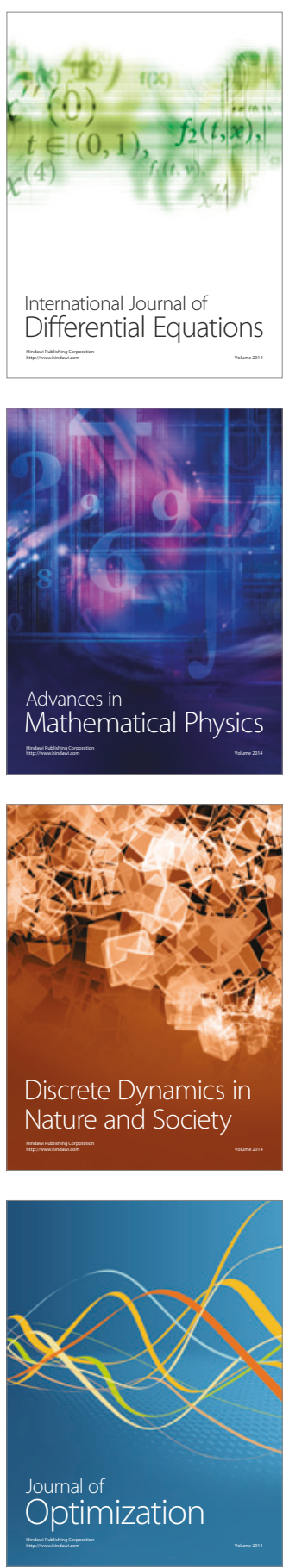